
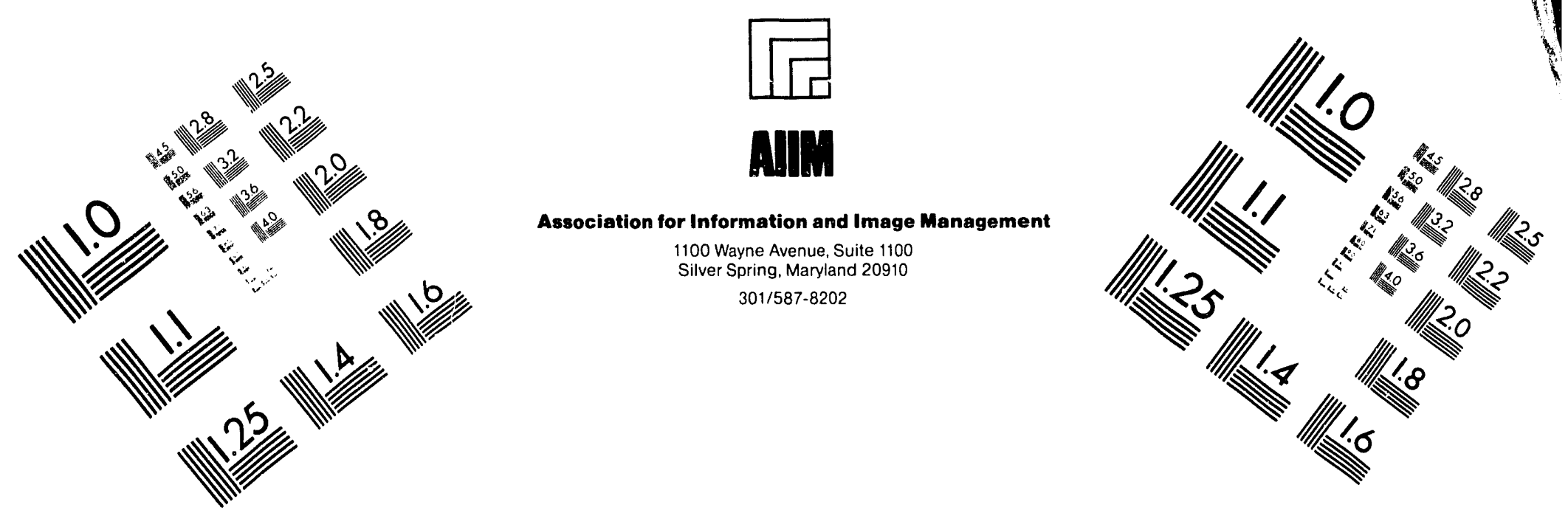

\title{
Centimeter
}

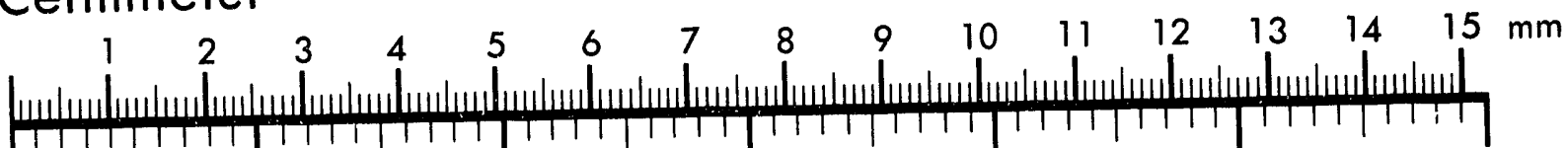

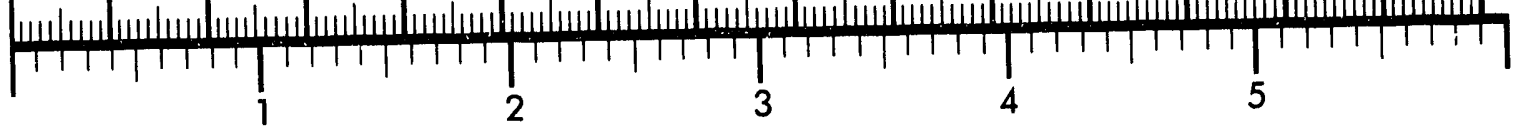
Inches
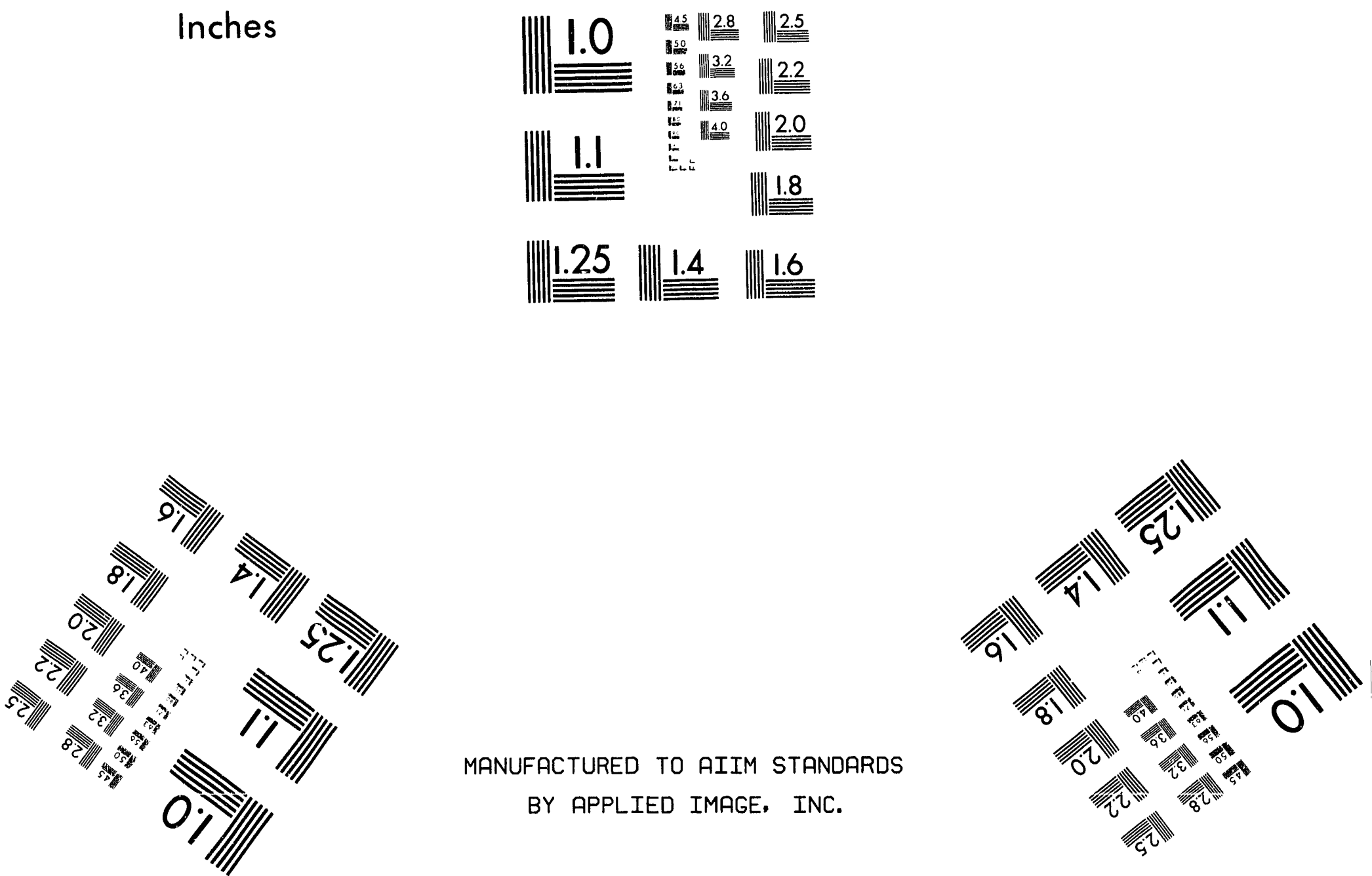

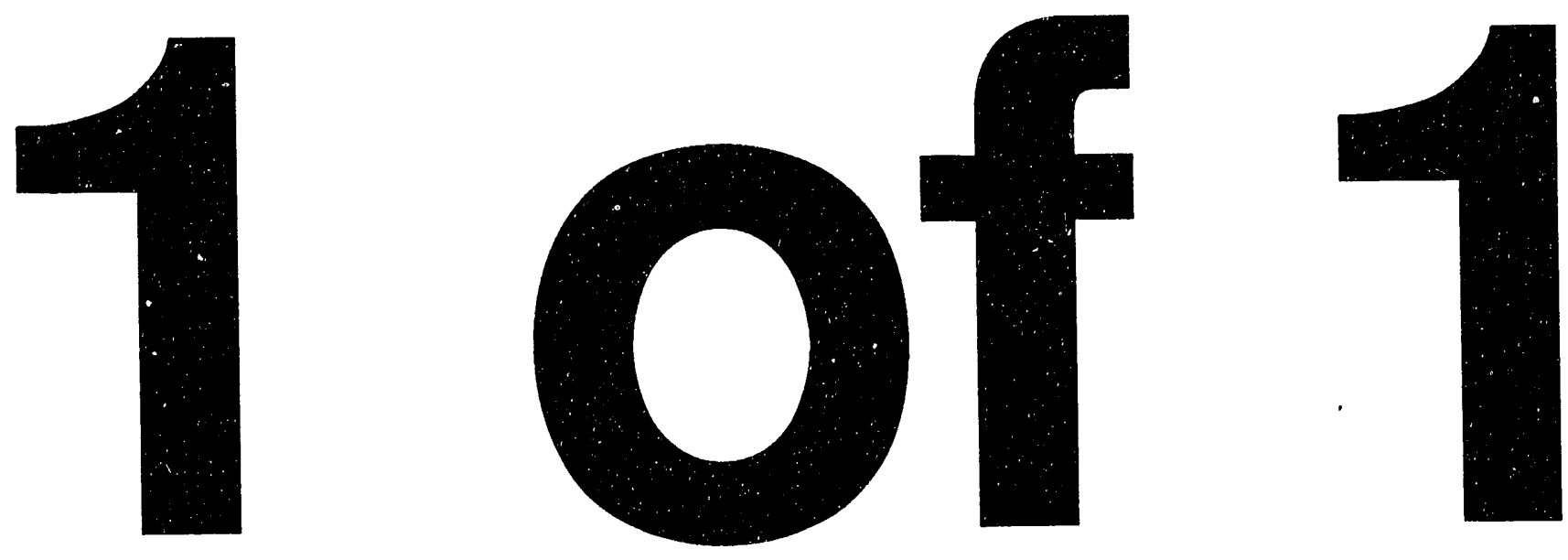


\section{Computer Simulation of Macromolecular Crystals and Their Defects}

Abstract of a lecture by Bernhard Wunderlich of work carried out in collaboration with
Bobby G. Sumpter, Donald W. Noid, and Guanghe L. Liang

Chemistry Division, Oak Ridge National Laboratory, Oak Ridge, TN 37831-6182, USA, and Department of Chemistry, The University of Tennessee, Knoxville, TN 37996-1600, USA

In this lecture I want to review ${ }^{1}$ computational results on the dynamics of polyethylene, derived from our research group during the last five years ${ }^{2}$ and connect them to experimentally known facts. It could be demonstrated that conformational defects can be created at temperatures as much as $100 \mathrm{~K}$ below the melting point and that the concentration continues to increase exponentially with temperature, ultimately leading to disordered crystals along the polymer chains (high temperature CONDIS crystals ${ }^{3}$ ). Although the rate of formation of these defects is high, approximately $1 \times 10^{10} \mathrm{~s}^{-1}$ at $350 \mathrm{~K}$, the defects do not, by themselves, lead to macroscopic motion that could give rise to lamellar thickening or deformation. The diffusion mechanisms involve coupling of large-amplitude torsional motion with transverse and longitudinal vibrations of the crystal, which lead to the formation of dispirations and twists. Such defects can, under proper conditions, move towards the end of the crystal, thereby causing a chain diffusion process that is probably at the root of lamellar thickening and deformation processes. Collective twisting of the chains without major influence of conformational defects leads to hexagonal or pseudo-hexagonal structures of the asymmetric motifs, involving dynamic multidomain arrangements of the chains. The especially efficient molecular dynamics simulation-code ${ }^{4}$ developed for this research produces reasonable agreement with experimental data on density, defect concentration, heat capacity, vibrational spectra (including stress-induced frequency shifts), melting temperature, and speed of sound. The simulations produced data on crystals of up to 30,000 atoms for times of up to 100 picoseconds $\left(10^{-10} \mathrm{~s}\right)$. The total simulation efforts needed a massive effort of approximately 8,000 hours of supercomputer CPU time between 1988 and 1993. Further progress with the next generation of parallel computers installed in our institutions awaits the development of advanced molecular dynamics simulation codes.

\footnotetext{
T For an extensive review see: Sumpter BG, Noid DW, Liang L, Wunderlich, B (1993/4) Atomistic Dynamics of Macromolecular Crystals, Adv Polynter Sci. to be published

2 Sumpter BG, Noid DW, Wunderlich, B (1990) Computer Experiments on the Internal Dynamics of Crystalline Polyethylene, Mechanistic Details of Conformational Disorder, J Chem Phys 93:6876; (1992) Computational Experiments on the Motion and Generation of Defects in Polymer Crystals, Macromolecules 25:7247

3 Wunderlich B, Möller M, Grebowicz J, Baur H (1988) Conformational Motion and Disorder in Low and High Molecular Mass Crystals, Adv Polym Sci 87

4 Noid DW, Sumpter BG, Wunderlich B, Pfeffer GA (1990) Molecular Dynamics Simulations of Polymers: Methods for Optimal Fortran Programming, J Comp Chem, 11:236; Noid DW, Sumpter BG, Cox RL (1991) Computational Strategies for Molecular Dynamics Simulations of Polymer Processes:
} Numerical Integration Schemes, J Comp Polym Sci 1:161

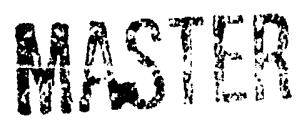

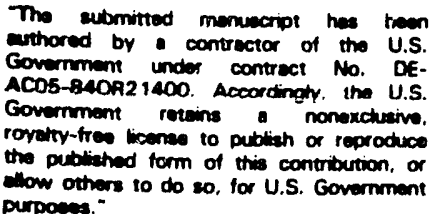

enthored by a contrector of the U.S. Covernment undes contrect No. DEAccordingty. the U.S.

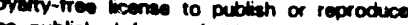

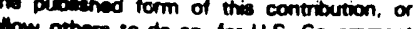
purpones." 


\title{
Computer Simulation of Macromolecular Crystals and Their Defects
}

\author{
Bernhard Wunderlich, Bobby G. Sumpter, Donald W. Noid, and Guanghe L. Liang \\ Chemistry Division, Oak Ridge National Laboratory, Oak Ridge, TN 37831-6182, USA, and \\ Department of Chemistry, The University of Tennessee, Knoxville, TN 37996-1600, USA
}

\begin{abstract}
Computational results on the dynamics of polyethylene, derived by our research group during the last five years are reviewed and connected to experimentally known facts. It could be demonstrated that conformational defects can be created at temperatures as much as $100 \mathrm{~K}$ below the melting point and that the concentration continues to increase exponentially with temperature, ultimately leading to disordered crystals along the polymer chains (high temperature CONDIS crystals). Although the rate of formation of these defects is high, approximately $1 \times 10^{10} \mathrm{~s}^{-1}$ at $350 \mathrm{~K}$, the defects do not, by themselves, lead at low temperature to macroscopic motion that could give rise to lamellar thickening or deformation. The diffusion mechanisms involve coupling of large-amplitude torsional motion with transverse and longitudinal vibrations of the crystal, which lead to the formation of disclinations, dispirations, and twists. Such defects can, under proper conditions, move towards the end of the crystal, thereby causing a chain diffusion process that is probably at the root of lamellar thickening and deformation processes. Collective twisting of the chains without major influence of conformational defects leads to hexagonal or pseudo-hexagonal structures of the asymmetric motifs, involving dynamic multidomain arrangements of the chains. The especially efficient molecular dynamics simulation-code developed for this research produces reasonable agreement with experimental data on density, defect concentration, heat capacity, vibrational spectra (including stress-induced frequency shifts), melting temperature, and speed of sound. The simulations produced data on crystals of up to 30,000 atoms for times of up to 100 picoseconds $\left(10^{-10} \mathrm{~s}\right)$. The total simulation efforts needed a massive effort of approximately 8,000 hours of supercomputer CPU time between 1988 and 1993.
\end{abstract}

\section{INTRODUCTION}

Much of the understanding of modern science is based on the microscopic description of matter. The length scale in question is the nanometer $(\mathrm{nm})$ or ångstrom $(\AA)$, distant from our macroscopic senses by at least a factor of $10^{4}$. Although it is well known, that the small-amplitude molecular vibrations, measurabie by infrared spectroscopy, have a frequency of up to $10^{14} \mathrm{~Hz}$, it has only recently become possible to follow the largeamplitude motion by realistic molecular dynamics simulation. These simulations are done with supercomputers and enable the extremely slow motion presentation that is needed to follow the fate of atoms and molecules on their movement in the solid state. The surprising result was that such large-amplitude motion can have an atomic time-scale in the picosecond range $\left(10^{-12} \mathrm{~s}\right)$, slower by only a factor $1 / 100$ than vibrations, but faster by a factor of $10^{9}$ than the fastest direct human observation (assumed to be milliseconds). It is thus a much more difficult task to mentally bridge the microscopic, atomic time-scale to the macroscopic, human time-scale than to bridge the corresponding length scales. Experimental verification of such fast large-amplitude motion is seen presently in the interpretation of solid state NMR results, as well as quasi-elastic neutron scattering. 
In this paper the first results from a five-year effort of molecular dynamics simulation of polyethylene crystals is discussed, with a special effort to establish a connection to the available ererimental results. These simulations have led to the identification of the mechanics of formation of conformational defects, twists, disclinations, and dispirations of chains, and crystal domain structures. Overall, a new view of the defect solid state of polymers has become possible. A more detailed discussion with close to 150 references has been prepared recently [1]. The reader is directed there, and at the limited key references repeated in this paper for an entry to the fast-growing literature in this field.

\section{MOLECULAR DYNAMICS SIMULATION OF CRYSTALS}

The molecular dynamics method (MD) involves the numerical solution of Hamilton's equations of motion, starting from some chosen initial positions and velocities of the atoms or particles of the system. In our highly efficient MD simulations, the integrations of the equations of motion are carried out in Cartesian coordinates, thus giving an exact definition of the kinetic energy and coupling [2]. Integrations are performed using a up to $12^{\text {th }}$ order predictor/corrector routine with variable time steps. This method (ODE) allows the integrations to be performed to a high accuracy, permitting conservation of all constants of motion to as many as four digits without the need for any ad hoc scaling of the momenta (total energy, angular and linear momenta).

The Hamiltonian is composed of the kinetic and potential energy for the system:

$$
\begin{aligned}
H-\sum_{n-1}^{m}\left[\sum_{i-100(n-1)+1}^{100 m} \frac{p_{x_{i}}^{2}+p_{y_{i}}^{2}+p_{z_{i}}^{2}}{2 M}+\sum_{i-100(n-1)+1}^{100 m-1} V_{\text {bond }}\left(i_{i, i+1}\right)\right. \\
\left.+\sum_{i=100(n-1)+1}^{100 m-2} V_{\text {bend }}\left(\theta_{i, i+1, i+2}\right)+\sum_{i-100(n-1)+1}^{i-100 m-3} V_{\text {introd }}\left(\tau_{i, i+1, i+2, i+3}\right)\right] \\
+\sum_{i=1}^{100 m}\left[\sum_{j} V_{\text {nonbonded }}\left(R_{i, j}\right)+\sum_{k=1}^{100 l} V_{\text {nonbonded }}\left(R_{i, k}\right)\right]
\end{aligned}
$$

Equation (1) refers to a crystal of $m$ dynamic chains of $100 \mathrm{CH}_{2}$-groups each. The second brackets represent the nonbonded interactions among all dynamic molecules $i$ with $j$, where the latter are the dynamic atoms not considered in Eqs. $(2,4$, and 5$)$ for the given $i$. The last term, summing over the interactions of the atoms $i$ with $k$, is only needed if the crystal is enclosed with $l$ chains of rigid (nondynamic) atoms, fixed to keep the rolume constant. The potential functions for the adjacent, bonded atoms $(i$ and $i+1)$ in $\mathrm{kJ} / \mathrm{mol}$ are:

$$
V_{\text {bond }}(r)-334.72\left(1-\mathrm{e}^{-19.9(\mathrm{r}-0.153)}\right)^{2}
$$

where $0.153 \mathrm{~nm}$ represents the equilibrium bond length (Morse equation). The interaction between any two nonbonded atoms is represented by the Lennard-Jones expression (note that along the chain the atoms must be separated by a sequence of three atoms or more): 


$$
V_{\text {nonbonded }}(R)-1.9748\left[\left(\frac{0.4335}{R}\right)^{12}-\left(\frac{0.4335}{R}\right)^{6}\right]
$$

where $R$ represents the appropriate distance between $i$ and $j$ or $k$. For the bending of a three-atom, bonded sequence the potential is:

$$
V_{\text {bend }}(\theta)-65.061\left(\cos 113^{\circ}-\cos \theta\right)^{2}
$$

with $113^{\circ}$ representing the equilibrium bond angle. The torsional coordinate $T$ between four successive atoms is linked to the internal rotational potential:

$$
V_{\text {introu }}(\tau)-8.3704-18.4096 \cos \tau+26.78 \cos ^{3} \tau
$$

The parameters of Eq.(5) have been fitted to give a barrier height of $16.7 \mathrm{~kJ} / \mathrm{mol}$ and a gauche - trans energy difference of $2.5 \mathrm{~kJ} / \mathrm{mol}$. These potential energy functions have been demonstrated to yield good spectroscopic, thermodynamic, and kinetic data, as well as to provide the atomistic details of temperature-dependent phase transitions for crystalline polyethylene and correspond to a collapse of the $\mathrm{CH}_{2}$-groups into a single particle of mass 14 amu [1].

Models with explicit hydrogens have also been simulated, with appropriately modified Eqs. (1-5). Since such full simulation increases the number of atoms (and the computation time) considerably, only selected simulations have been done with explicit hydrogens [1]. Furthermore, as will be discussed in the next section, it is not possible to reproduce the ideal crystal structure with the present hydrogen force fields. Many other properties, in turn, can be represented as well, or better, and certainly faster, by the united atom model.

For all simulations, the initial conditions involve imparting a randomly chosen momentum, subject of a zero center-of-mass velocity constraint. Due to the large number of atoms in the system, and thus the high density of vibrational states, thermal equilibrium, as described by a Boltzmann distribution, is rapidly achieved. An effective temperature can then be determined from the average kinetic energy $<K E>$ by:

$$
\left\langle K E>-\frac{3}{2} N k T-\sum_{i=1}^{100 m} \frac{p_{x_{i}}^{2}+p_{y_{i}}^{2}+p_{z_{i}}^{2}}{2 M_{i}}\right.
$$

where $k$ is Boltzmann's constant, $T$ is the temperature, $p$ the Cartesian momentum and $M$ the mass of the atoms $i$. In general, analyses of the simulations were started after allowing the kinetic energy to redistribute.

The simulations produced data on crystals of up to 30,000 atoms for times of up to 100 picoseconds $\left(10^{-10} \mathrm{~s}\right)$. The total simulation efforts needed, thus, a massive effort of approximately 8,000 hours of supercomputer CPU time between 1988 and 1993 [1-3]. Further progress with the next generation of parallel computers, already installed in our institutions, awaits the development of advanced molecular dynamics simulation codes. Considerable time is expected to be needed to accomplish this task and to find the necessary financial support for this effort. 


\section{IDEAL CRYSTALS}

Helped by the high external symmetry, the understanding of crystals has always been at the forefront of the natural sciences [4]. Kepler, for example, explained the multiplicity of the shapes of snow flakes by the large number of possible regular packings of spheres some 200 years before the accepted proof of the atomic nature of matter by Dalton in 1808 . Figure 1 reproduces a modern-looking crystal, drawn in 1690 by Huygens. About 250 years later, the knowledge of crystals had advanced from the general principle, displayed in Fig. 1, to the detailed, ideal structures determined by X-ray diffraction. The structure of orthorhombic polyethylene (Pnam) is shown in Fig. 2. The projection along the $c$-axis places the zig-zag chains at the corners of the unit cell and in the center. Following the

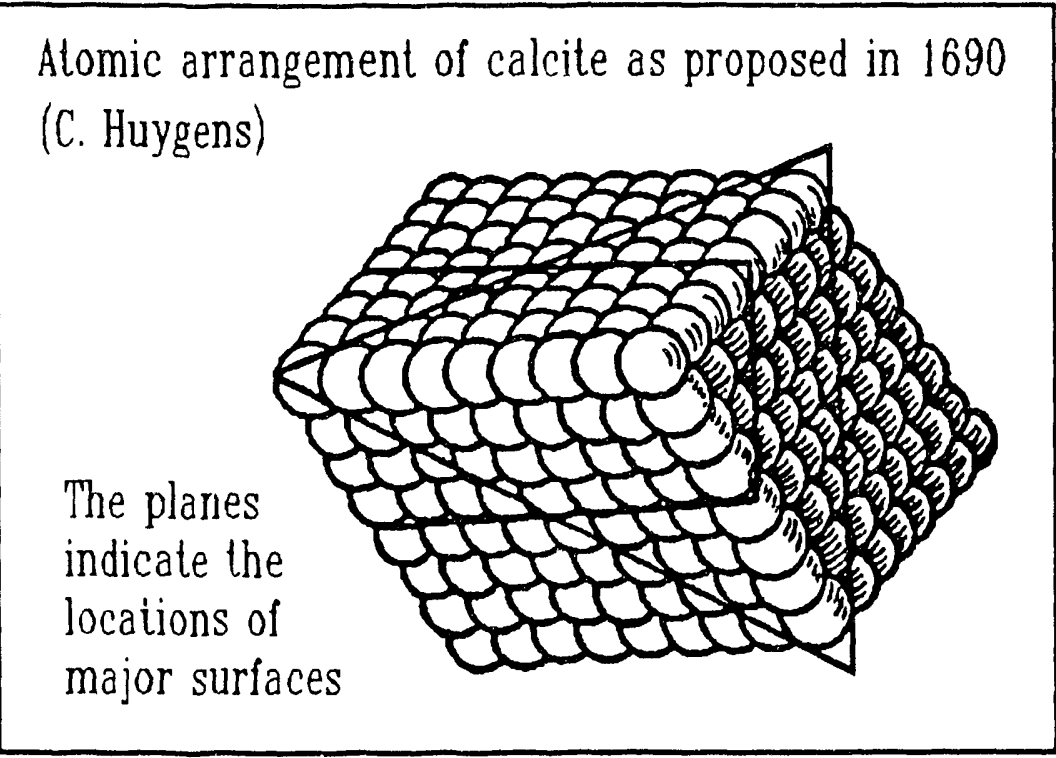

Figure 1 packing rules developed by Kitaigorodskii [5], and typical van der Waals radii, one can derive that Pnam is, indeed, the densest possible crystal with a coordination number of six for the chains, and a packing fraction $k$ of 0.70 ( $k=$ van

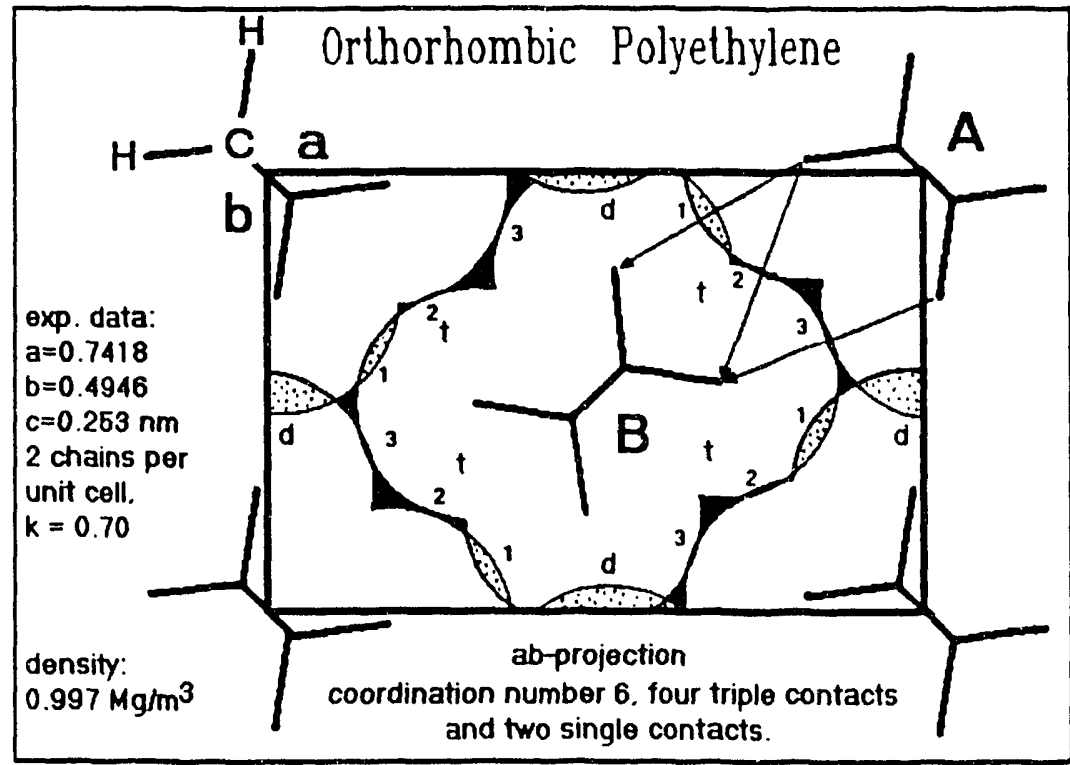

Figure 2 der Waals volume / unit cell volume). Four of the neighboring chains show strong triple-contacts (marked by arrows in Fig. 2), the other two have only single contacts. The structure is thus critically dependent on packing the $\mathrm{CH}_{2}$-groups of the center chain into the appropriate depressions of the surrounding chains.

In such analyses, it was discovered that, in order to get agreement with experimental lattice parameters, an artificially large, lateral van der Waals radius of $0.135 \mathrm{~nm}$ had to be used (instead of the commonly accepted value of $0.117 \mathrm{~nm}$, derived from contacts in the bond direction). Without this correction, the packing fraction turned out to be 0.85 [4]. The large variation in van der Waals radius with direction is unique with hydrogen and has been attributed by Bunn [6] to a nonspherical shape of the covalently bound hydrogen. 
Naturally, the packing of hydrogen in polymer crystals is of central importance and has not yet been resolved.

Our first MD simulations with explicit hydrogens used a spherical van der Waals interaction, employing an expression similar to Eq. (3) [1]. As should perhaps have been expected, the resulting crystal structure had an excessively high density (and preferred a somewhat different structure). The higher density resulted in reduced conformational defect concentrations. At present, one must thus be cautious in the interpretation of all MD simulations involving nonbonded interactions with explicit hydrogen atoms. By clearly identifying the problem of the nonspherical hydrogen shape, it is hoped that progress in solving the hydrogen shape will be forthcoming soon, although a more complicated Eq. (3) for the nonbonded interaction will, again, increase the computation time.

The united atom force

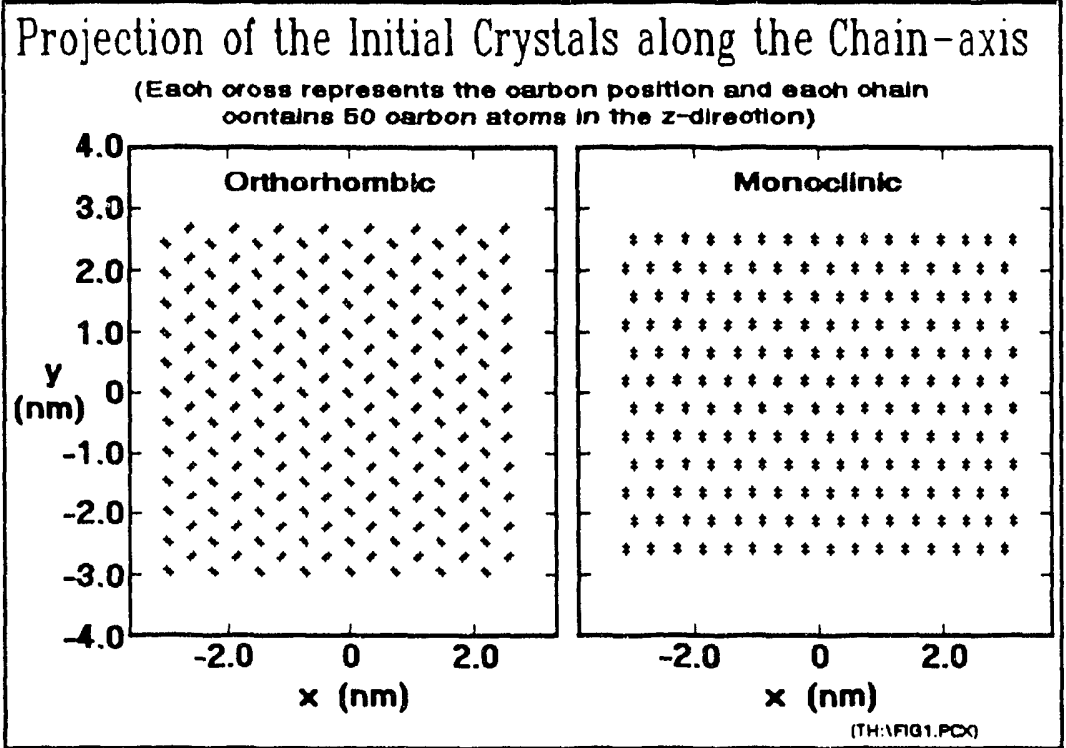

Figure 3 fields of Eqs. $(2-5)$, in turn, are well chosen and produce crystals with close to experimental densities. Since the united atom lacks the detailed mm2 point symmetry of the $\mathrm{CH}_{2}$-group, indicated in Fig. 2, the

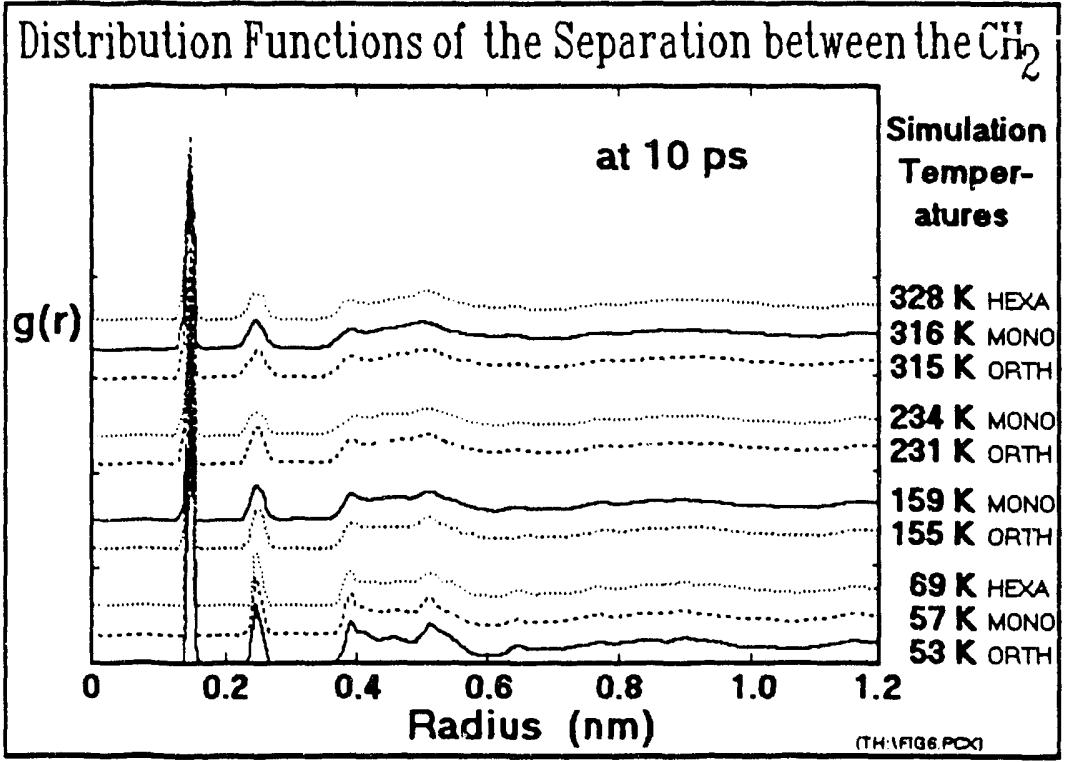

Figure 4 resulting crystal structure is hexagonal and can serve as a mode! of the high-temperature (and -pressure) form of polyethy:ene. The orthorhombi: structure, can, however, also be simulated by enclosing the dynamic chains in a ring of orthorhombically placed rigid chains (constant volume simulation). Figure 3 shows two of the initial ideal crystal structures chosen for simulation $(\mathrm{ORTH}=$ orthorhombic, as in Fig. 2, and MONO $=$ monoclinic). $\mathrm{A}$ third one, included a random placement of the zig-zag chains, to simulate a hexagonal structure (HEX). The size of each crystal was initially about $6.0 \times 6.0 \times 6.3=227 \mathrm{~nm}^{3}$. This is sufficiently large to serve as a model for polymer crystals, which are typically between 2 and $20 \mathrm{~nm}$ thick. The simulations were conducted in a temperature range from 55 to $410 \mathrm{~K}$ for 10 to 100 ps with the nonbonding potential represented by Eq. (3), being 
cut-off at $1.0 \mathrm{~nm}$ to conserve computation time. The crystals quickly reached a steady-state, enabling us to compare dynamic and structural behavior with experimental results. After about $4 \mathrm{ps}$, the radial distribution functions ceased to be dependent on time and became indistinguishable among the different initial structures, which means that all three starting structures (ORTH, MONO, and HEXA) had been transformed to the same "steady-state", as can be seen from the radial distribution curves of Fig. 4 .

Once the crystal structure is established, its properties can be assessed. Figure 5 shows a density plot. Oscillations occur about the average, which is close to the experimental density of polyethylene. At about the same temperature, different initial structures have slightly different density fluctuations in direction and amplitude, but the same frequency $\left(\approx 3 \times 10^{11}\right.$ $\mathrm{Hz}$ ). Assuming that one sees the first fundamental vibration of the crystal with a wavelength of about $12 \mathrm{~nm}$ (twice the crystal size), one can estimate the speed of the wave to be about $4 \mathrm{~km} / \mathrm{s}$, which, in turn, is typical for measurements of the speed of sound in polymers.

Once a crystal structure has been established, it is thus possible to establish its properties. A number of these, such as the just illustrated density and speed of sound, as well as modulus and heat

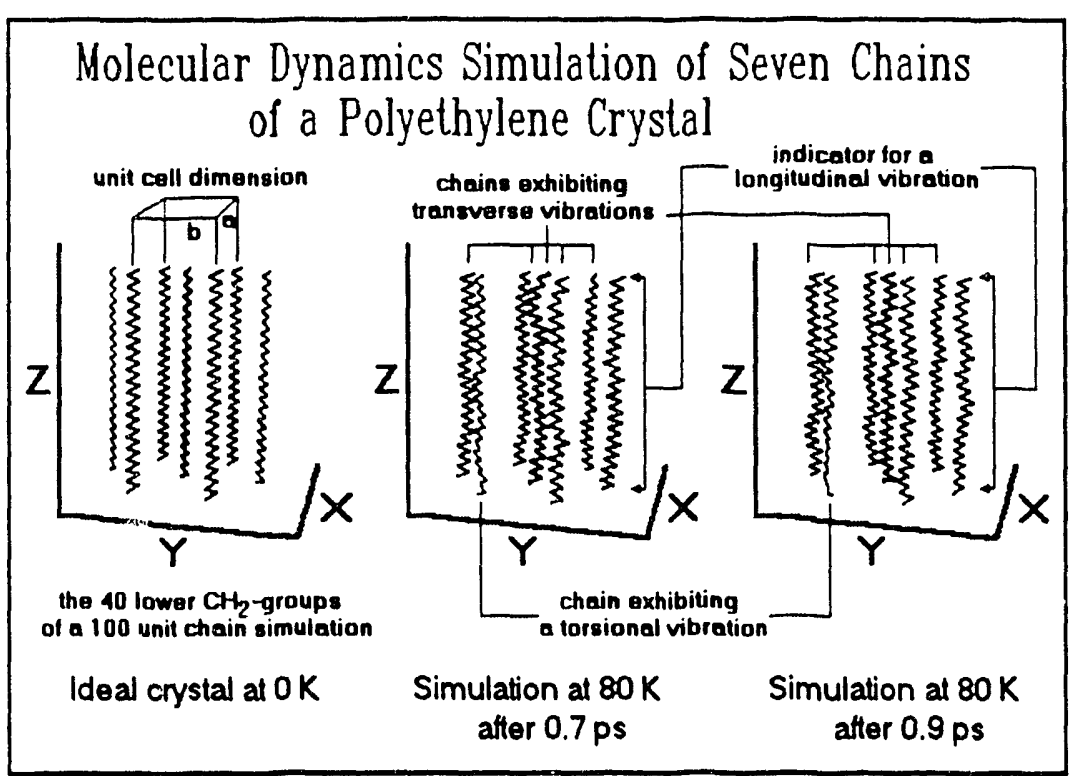

Figure 6

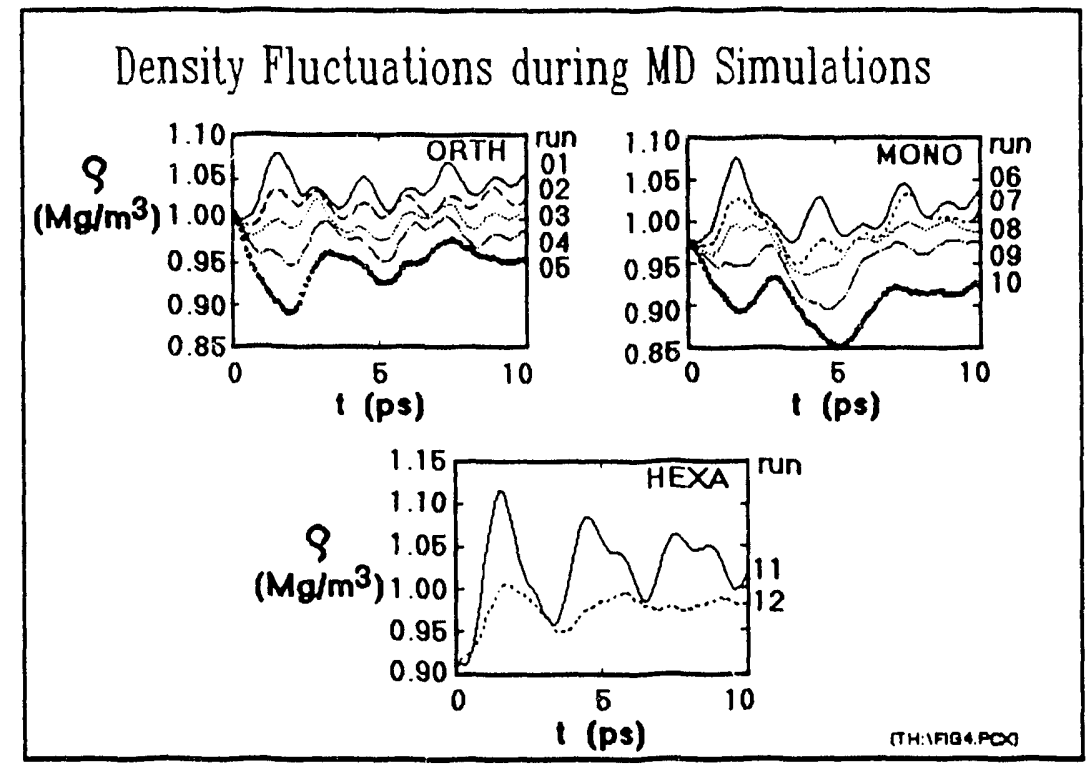

Figure 5 Simulation temperatures of the runs: $1,53 \mathrm{~K} ; 2$, $155 \mathrm{~K} ; 3,231 \mathrm{~K} ; 4,315 \mathrm{~K} ; 5,410 \mathrm{~K} ; 6,57 \mathrm{~K} ; 7,159 \mathrm{~K} ; 8$, $243 \mathrm{~K} ; 9316 \mathrm{~K}, 10,403 \mathrm{~K} ; 11,69 \mathrm{~K} ; 12,328 \mathrm{~K}$. content, agree well with the ideal crystal structure. These properties are called structure insensitive, to contrast with the structure sensitive properties (such as the ultimate strength, stress for plastic deformation, chemical reactivity, and diffusivity).

The thermal properties of macromolecules can, next, be derived from the vibrational behavior. The motion of a polyethylene crystal, simulated at constant volume and at low temperature, is shown in Fig. 6. It is easy to see the three basic, 
skeletal vibrations that are coupled over several $\mathrm{CH}_{2}$-groups along the chain: transverse, torsional, and longitudinal vibrations.

The quantitative analysis of simulations of this type is able to reproduce the full frequency spectrum of a polyethylene crystal [1]. The vibrational spectrum, in turn, is easily linked to the heat capacity by summing the contribution of each vibrational frequency to the heat capacity using an Einstein function [7]:

$$
C_{v} / N R-\mathrm{E}\left(\Theta_{\mathrm{B}} / T\right)-\frac{\left(\Theta_{\mathrm{B}} / T\right)^{2} \exp \left(\Theta_{\mathrm{E}} / T\right)}{\left[\exp \left(\Theta_{\mathrm{E}} / T\right)-1\right]^{2}}
$$

where $N$ represents the multiplicity of the vibration and $\theta_{\mathrm{E}}$ is the Einstein temperature (frequency), expressed in $h v / k$. Once the heat capacity is known from absolute zero of temperature, the enthalpy, entropy, and Gibbs energy can easily be calculated. Our ATHAS data bank [8] has been used over the last twenty years to accomplish this correlation for more than 100 polymers. Figure 7 shows the example of polyethylene. The skeletal vibrations, indicated in Fig. 6, and the additional group vibrations, consisting mainly of $\mathrm{C}-\mathrm{H}$ motion that can be simulated with explicit hydrogen models, make up the heat capacity at constant volume $C_{\mathrm{v}}$. After conversion to heat capacity at constant pressure, $C_{\mathrm{p}}$, the comparison with experiment shows agreement from 0 to $300 \mathrm{~K}$.

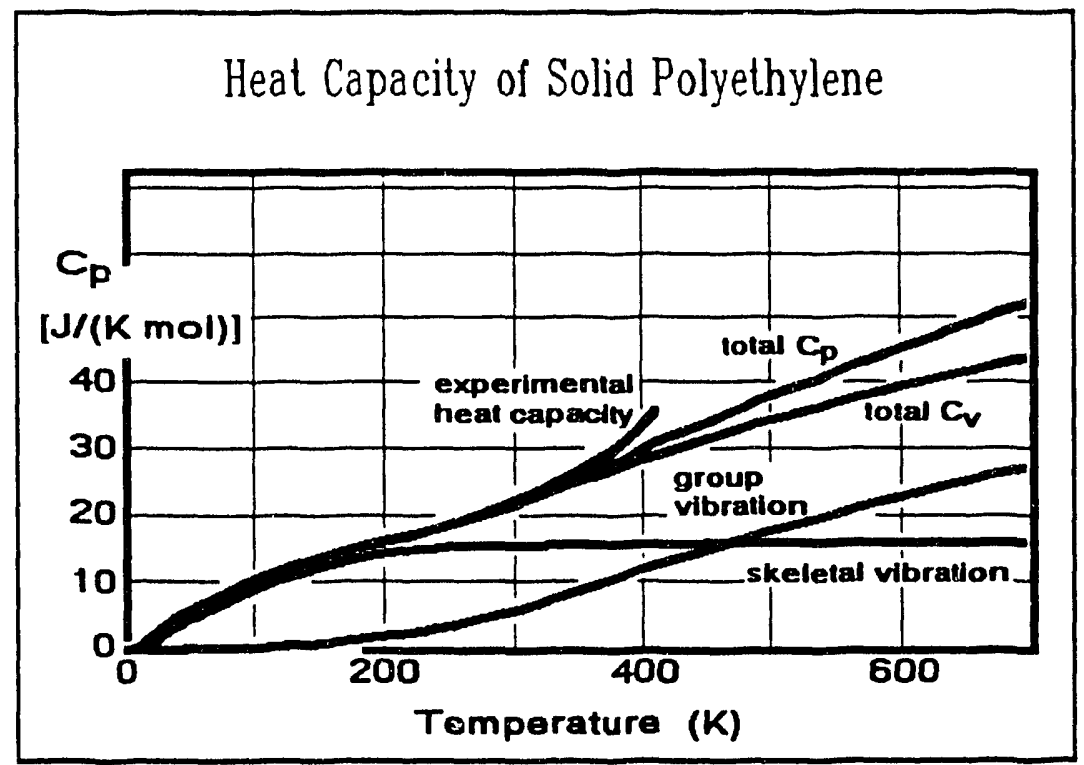

Figure 7

Not only can the heat capacity, and with it also all integral thermodynamic functions, be established with an approximate frequency spectrum, it is also possible to predict heat capacities of polymers with similar chemical structures and establish heat capacities for copolymers in the solid state. Similar results are expected for all other structure-insensitive properties.

At about $300 \mathrm{~K}$ the experimental heat capacity starts to deviate significantly from the calculation that was carried out under the assumption that only vibrations govern the thermal energy. This is a first indication of defect contribution to the thermal properties and will be discussed in the remaining part of this paper.

\section{GENERAL DESCRIPTION OF DEFECTS IN POLYMER CRYSTALS}

To describe semicrystalline polymers, a two-phase model has been commonly used over the last 50 to 60 years. One of the two phases being represented by perfect crystals, the other 
by a fully amorphous phase. Structure-insensitive properties vary approximately linearly with the weight or volume fraction of this crystallinity. Since such a two-phase structure in a one-component system can, according to the phase rule, not be in equilibrium, there must be constraints hindering further crystallization of the amorphous phase, i.e. there must be a connection between the two phases (microphases). Because of this link between crystal and amorphous phase, the latter has also been called a three-dimensional, "amorphous defect" [4]. Its structure and mobility is still not well understood, but may in the future be amenable to MD simulations of the type presented here.

Characteristic for polymeric materials are also two-dimensional defects, represented by surfaces. External surfaces are always the prime defect of a single crystal. For polymer crystals they are particularly important because of the usually small crystal size. The highly regular surfaces of solution-grown crystals could be analyzed in detail by optical and electron microscopy, and more recently also by atomic force microscopy. These investigations gave much information about chain folding, tie molecules, fold-sector boundaries, stacking faults, twin boundaries, kink bands, etc.

Figure 8 illustrates MD simulations of the hexagonal crystal. One can see domains of different orientation of the $\mathrm{CH}_{2}$-zig-zag planes (setting angle). Even at $57 \mathrm{~K}$ (Fig. 8a) the crystal structure deviates from perfect order (compare to Fig. 3 ). Not only are the projections of the chains broadened by vibrations, but one can also see a break-up into at least two domains, separated by boundaries of two to three

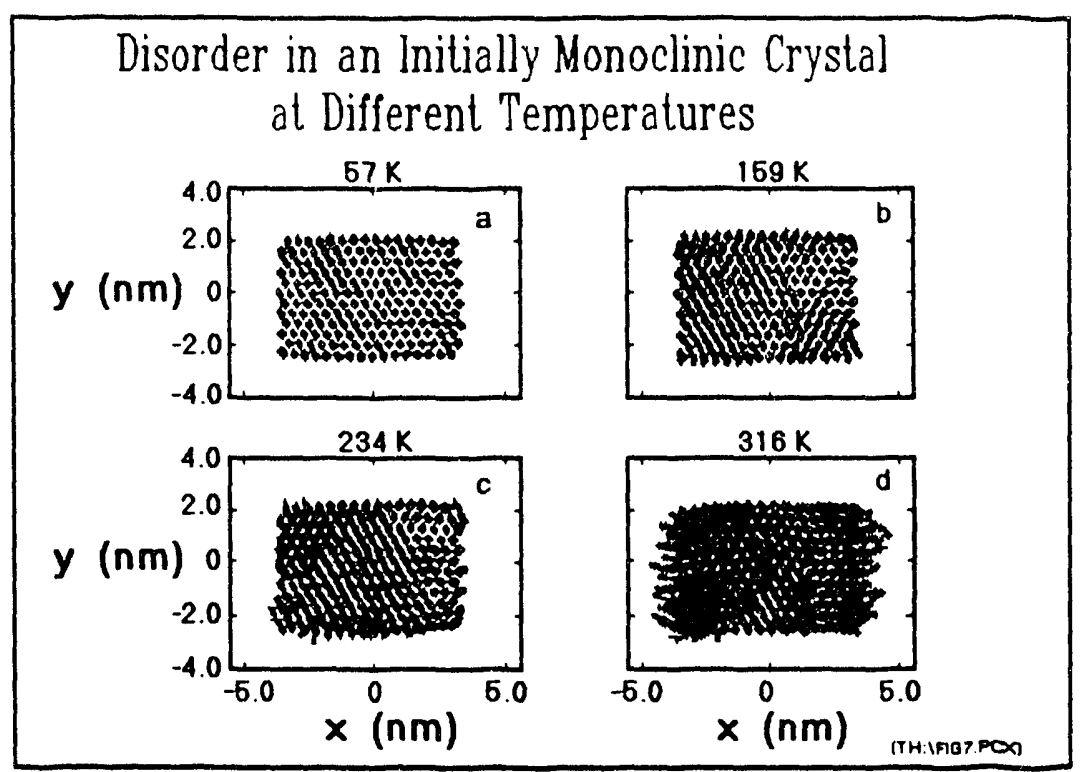

Figure 8 Structures after 10 ps simulations. layers of chains. At $234 \mathrm{~K}$ the crystal becomes more disordered and develops easily recognizable domains (Fig. 4c). The disorder increases with temperature and becomes significant at $316 \mathrm{~K}$ where, in addition, a "fuzzy" surface structure is evident (Fig. 4d). This occurs at a temperature that is still about $50 \mathrm{~K}$ below the experimental melting point. The scale of disordering is not uniform throughout the entire crystal, but seems to vary from one domain to another. It will be shown, below, that these nanometer-size domains are dynamic and change orientation and boundaries on a picosecond time scale, providing the average needed to fit an overall hexagonal symmetry.

Decreasing the dimensir nality of the defects leads one to dislocations (one-dimensional defects). The classical edge- and screw-dislocations of crystals of small molecules could also be documented in crystals of macromolecules. Particularly obvious are growth spirals with a Burgers vector of the size of the lamellar thickness, i.e. 5-50 nm, which are produced during crystal-growth [4]. Dislocations with a Burgers vector of unit-cell dimensions were seen by electron microscopy with the help of moiré patterns and were linked to chain ends in the crystal. A connection to deformation processes, as was found 
in crystals of small molecules was not possible because of the sessile nature of many dislocations and the absence of a source for new defects during deformation.

The most important deformation process of polymer crystals is that occurring on drawing, cold-extrusion, and rolling. These processes involve a quite chaotic process, as is illustrated in Fig. 9. Based on the MD simulations the defects involved in these processes involve the conformational motion cou-

pled to skeletal vibrations, and fall into the class of point defects. They are much different for polymer crystals when compared to small-molecule crystals and provide the ultimate element in the understanding of structure-sensitive properties. The differences arise from the connectiveness of the permanent covalent bonds of the backbone chain. Vacancies can only arise together with other defects such as kinks, chain ends, or wrong substituents in the chain (copolymers). Interstitial atoms must be linked with chain disorder, copolymerization, or monomeric impurities. Similarly, substitutional positions must be caused by prior copolymerization or by cocrystallization with small molecules (rare) [4]. Chain disorder, such as kinks, folds, jogs, torsions (twists) are the basic point defects in macromolecular crystals [4].

A starting point of defect evaluation can be the rotational isomer model. It makes use of the three-fold minimum in rotational energy [Eq. (5)]. To produce a defect in an all-trans sequence of polyethylene, one can, for example, represent a defect conformation as a sequence of gauche $\left(\mathrm{g}^{+}, \mathrm{g}^{-} ; \tau=300^{\circ}\right.$ and $60^{\circ}$, respectively) and trans $\left(\mathrm{t} ; \tau=180^{\circ}\right)$ conformations. A simple defect in polyethylene is a "2g1 kink," represented by the sequence of $\mathrm{g}^{ \pm} \mathrm{tg}^{\mp}$. The symbol $2 \mathrm{~g}$ indicates the number of gauche conformations, and the second numeral, the amount of shortening of the chain in multiples of $c / 2$ ( $c$ is the unit cell dimension along the chain axis). A $2 \mathrm{~g} 1$ kink displaces the chain in addition by about 0.2 $\mathrm{nm}$ in the directions at right angles to the zig-zag plane. The latter displacements were proposed to be removed by distortion of the chain. In case the displacement are bigger, as in $2 \mathrm{~g} 2$, they reach a neighboring zig-zag plane and are called jogs. The intermolecular energy of a $2 \mathrm{~g} 1 \mathrm{kink}$ was estimated from the increased volume multiplied with the cohesive energy density. Overall, it was estimated that an equilibrium concentration of 1 kink per 250 carbon atoms should be possible at $400 \mathrm{~K}$ [9].

Defects that do not involve rotational isomers, but gradual twists, compressions or expansions of the chain have also been proposed earlier. Successive small rotations about the backbone bonds and bond angle deformations are involved in such defects. A $360^{\circ}$ rotation about the chain axis leads to proper register with the neighboring chains in the crystal above and below the defect. Defects of this type are called disclinations. Finally, a rotation of $180^{\circ}$ can be combined with a translational defect of $c / 2$ to achieve crystalline 
register above and below the defect. Such combination of disclination and dislocation is called a dispiration [10].

Before it became possible to simulate a sufficiently large crystal by $M D$, the detailed behavior and energetics of point defects was not well understood. The often applied molecular mechanics calculations to establish the lowest energy conformations are not able to map out the actual reaction path and often lead, when considering intermolecular interactions, to much too high energies and activation energies.

\section{SIMULATIONS OF POINT DEFECTS}

The immediate observation on MD simulation, even for the case of a single chain surrounded by fixed chains, was that more than $100 \mathrm{~K}$ below the expected melting temperature a large number of conformational defects could be seen. Based on molecular mechanics calculations, it was deduced that such defects should be rare. Rotating a single bond from trans to gauche should completely remove the ability to fit into the array of parallel chains of the crystal. Figure

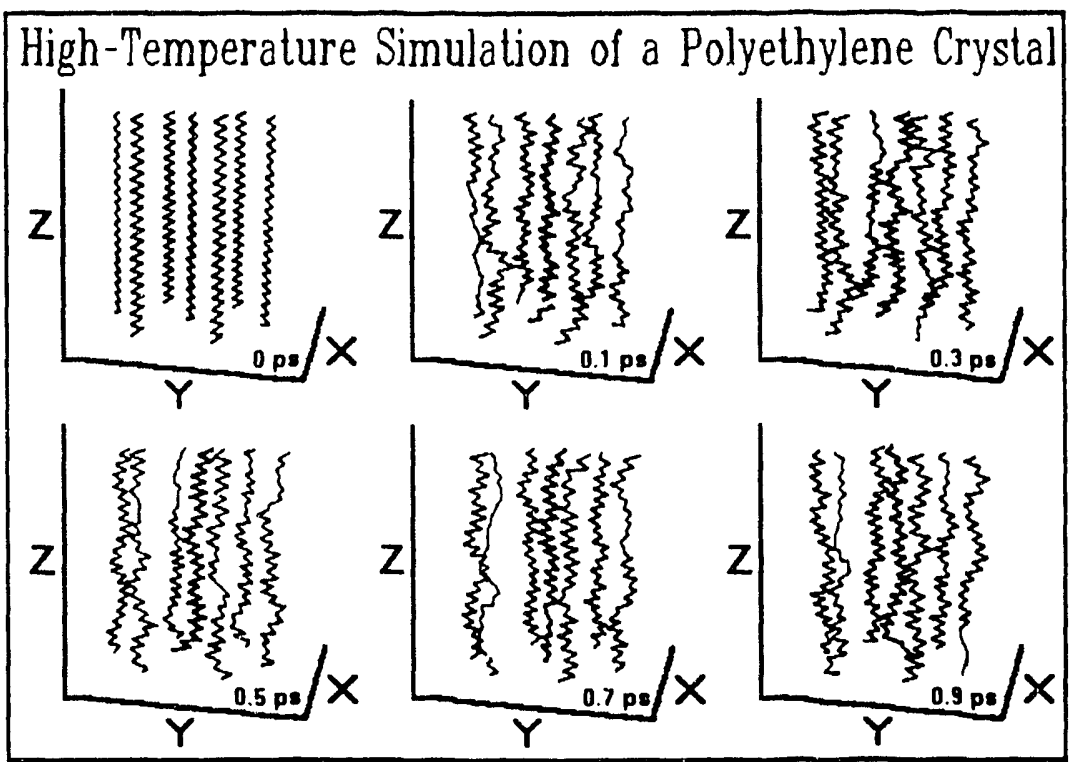

Figure 10 10 illustrates how such disorder is spread over larger areas of the crystal with torsional and bending vibrations being involved in the existence of the conformational defects (compare to Fig. 6). Figure 10 represents results from simulations above actual melting. Due to the presence of a static

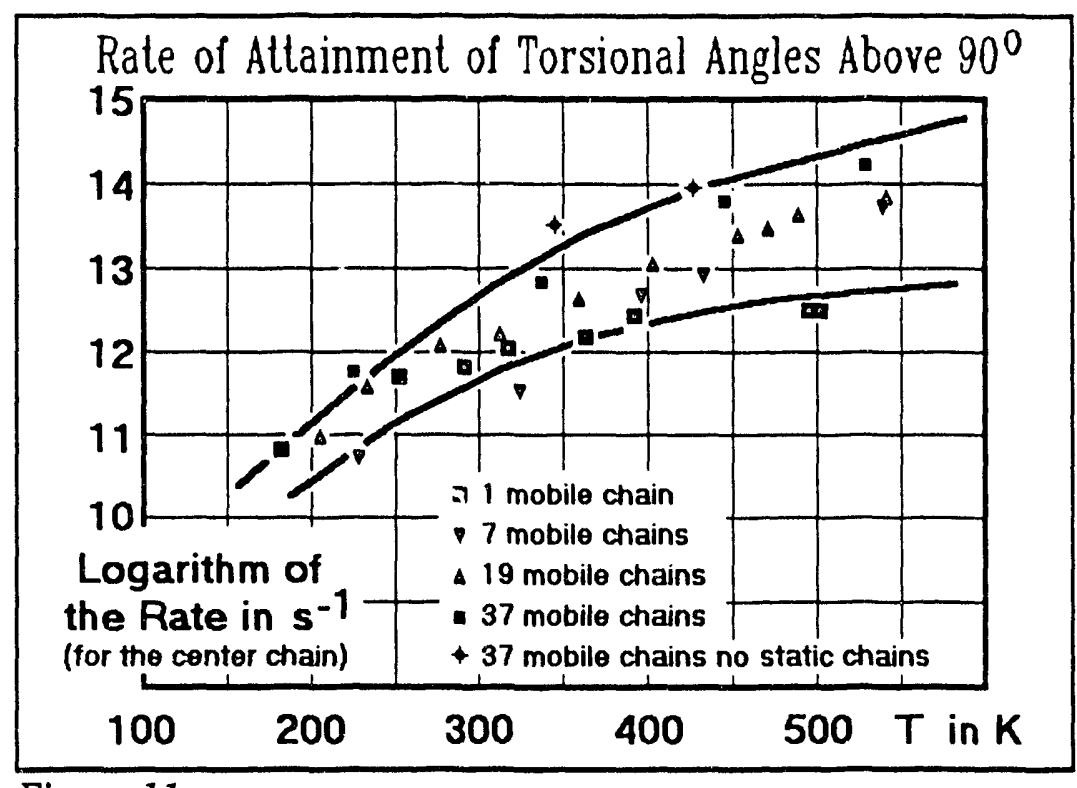

Figure 11 ring of 12 additional chains that maintain a constant volume, melting is avoided. The short-time simulation can in this way show several defects.

The rate of formation of gauche-defects (counted as soon as the rotation angle $\tau$ exceeded $\pm 90^{\circ}$ from the trans conformation) as a function of the size of the model crystal and temperature is displayed in Fig. 11. As the number of surrounding chains increases, the restriction due to the rigid 
crystal decreases, and the rate of formation of gauche bonds increases by 0.5 to 2.0 orders of magnitude. The upper curve represents a limit of no enclosure of rigid chains (constant, pressure simulation). At $350 \mathrm{~K}$ there are on the order of $10^{12}$ large, internal rotations per second about the backbone bonds, or about $10^{10}$ per bond. Such an enormously active source of defects can naturally drive many defect-linked processes in polymer crystals. From rate data, as shown in Fig. 11, the activation energies for the formation of gauche bonds could be derived using the transition state theory. It was found to be close to the potential energy barrier introduced in Eq. (5), varying from 13 to $25 \mathrm{~kJ} / \mathrm{mol}$, with 16.7 $\mathrm{kJ} / \mathrm{mol}$ most common for the less restrictive models. The relatively low steady state concentration of gauche conformations suggests an extremely short life-time of a gauche defect. So short, in fact, that experiments that average over long time periods do not observe the deviations.

The population of rotated bonds for the constant-volume, 19 mobile chains surrounded by 18 static chains, with each chain consisting of $100 \mathrm{CH}_{2}$ groups, is shown in Fig. 12. as a function of temperature. Adding the shallow peak about the gauche angle at $322 \mathrm{~K}$, for example, leads to a total concentration of only about $0.5 \%$. Note that for small deformation angles the (vibration) amplitudes are truncated heavily, with the actual percentages written at the top for each standard interval. Note also, that the crystal environment has changed the gauche maximum from $\cos \tau=0.5$ to $\cos \tau \approx 0.4$ (rotation from $180^{\circ}$ to $66^{\circ}$ or $294^{\circ}$ instead of $; 0^{\circ}$ or $300^{\circ}$ ). The increased potential energy due to the conformational defects was closel, duplicated by the observed increase in heat capacity in the same temperature range, as shown in Fig. 7 [3].

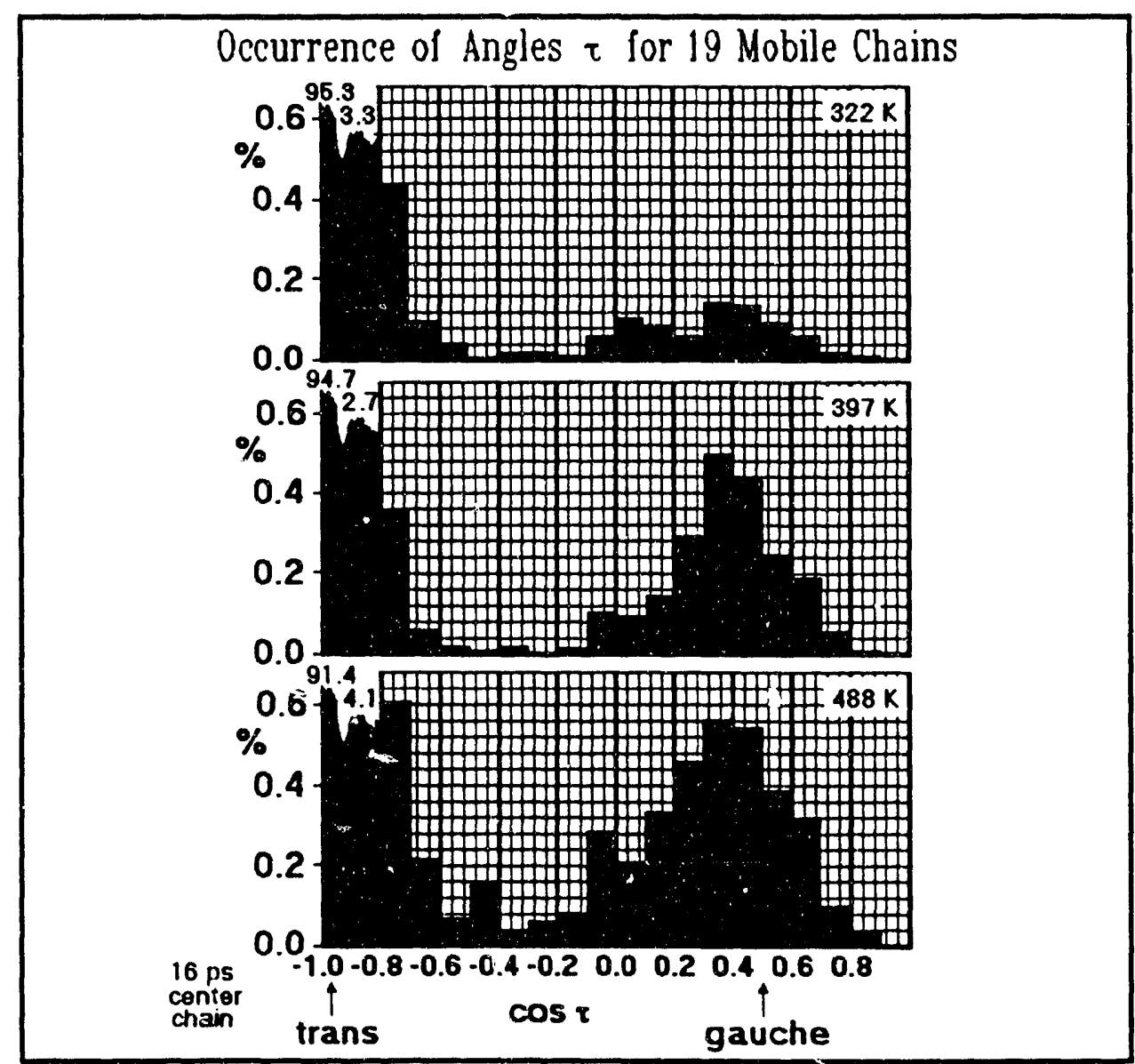

Figure 12 
Examination of the dynamics depicted, for example, in Fig. 10 enables the search for possible point defects and a microscopic mechanism for structure-sensitive macroscopic processes. Figure 13 shows a typical plot of the lie-time of gauche defects. The lifetime of the defects is, as expected in the picosecond range and shows predominant sequences that can be characterized as $\mathrm{g}^{ \pm} \operatorname{tg}^{\mp}$ kink defects and cause a shortening of the chain by $c / 2$, in addition to some displacement in the $a$ and $b$ crystallographic directions. From the measured average chain-length at 440 $\mathrm{K}$ there is a shortening of about $1 \mathrm{~nm}$, or an equivalent of four $2 \mathrm{~g} 1$ defects, in approximate agreement with Fig. 13.

Mechanistic details for the formation of a $2 \mathrm{~g} 1$ kink

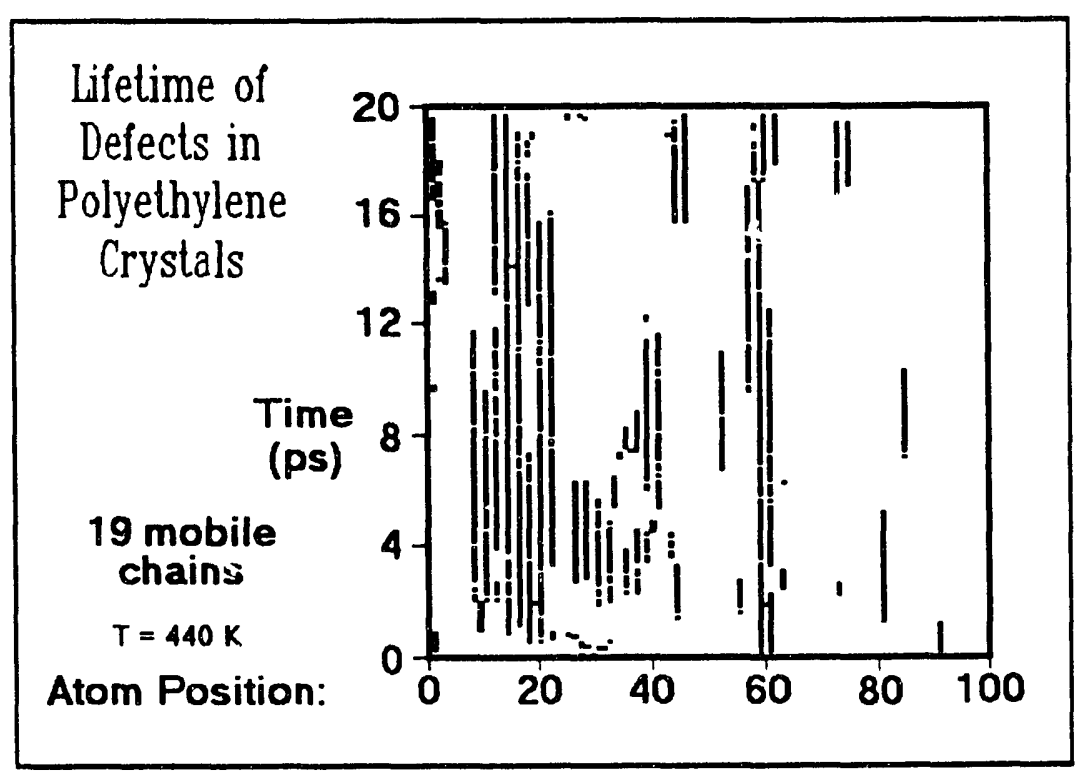

Figure 13 can be derived from Fig. 14

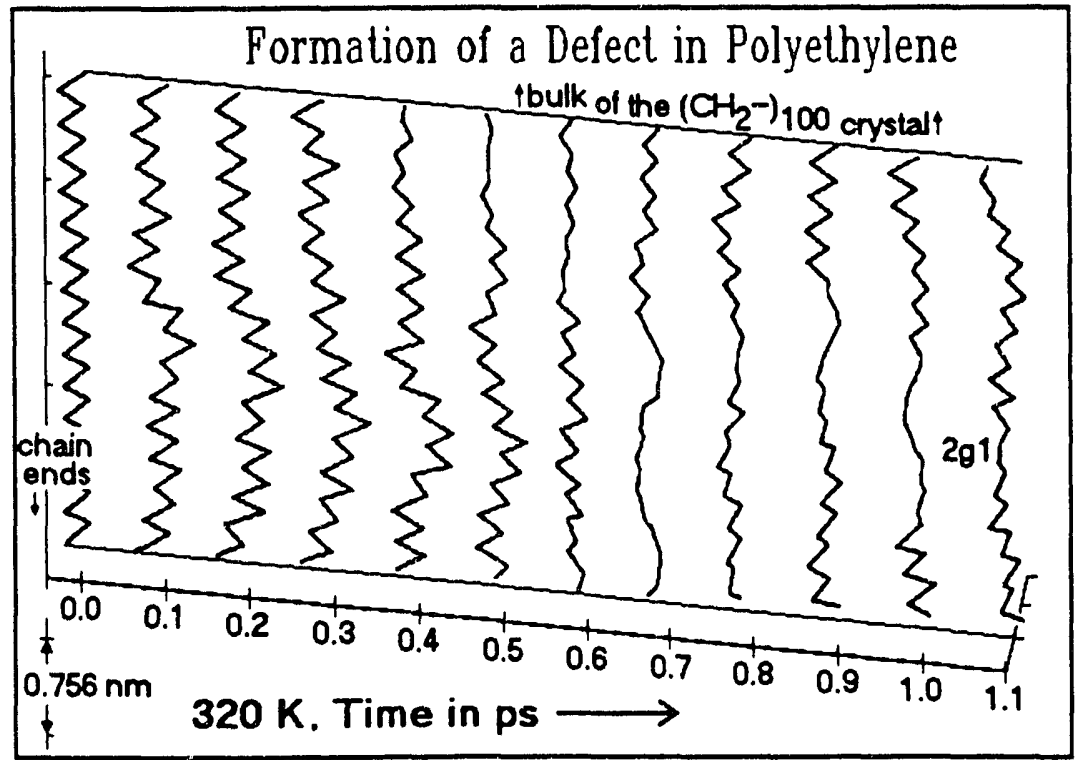

Figure 14

which depicts the conformation of a single chain as a function of time. The first 1.1 ps of a simulation at 320 $\mathrm{K}$ are displayed for the bottom portion of a chain in a crystal. One can easily recognize the process that leads in the end to the defect formation, a transverse vibration initiated at time $0.1 \mathrm{ps}$, a compression of the chain at 0.7 to $1.1 \mathrm{ps}$, and a torsional vibration moving into the field of view at time 0.5 ps. Their unique collision between 0.7 and 1.0 ps causes the $180^{\circ}$ turn of the chain end at $0.8 \mathrm{ps}$ and the indicated localized $2 \mathrm{~g} 1$ kink at 1.1 ps. The kink caused the remaining end of the chain to be in register $1 / 2$ unit cell length removed upwards. In this particular event, registered in Fig. 14, the life-time of the $2 \mathrm{~g} 1$ defect was about 2 ps [3].

Although the majority of bonds are at any one time close to trans "conformers", each of them is found to frequently deviate more than expected from normal vibrations. The zig-zag chains often obtain twists by sequential deviations in the same rotation direction. The formation of such twists is demonstrated in a spatial plot in Fig. 15. A series of time 
snap-shots at $316 \mathrm{~K}$ is given for the center-chain of a simulated crystal as described in Fig. 3. As can be seen, the chain undergoes rapid twisting motion that results in a change of the orientation of the zig-zag plane of the molecule. After about $1.5 \mathrm{ps}$ the chain is rotating via segmental motion, starting at the top of the crystal. Within about 0.5 ps the chain has turned by about $90^{\circ}$. Similarly, a new side-on view of the zig-zag develops between 7 and 8 ps, indicating

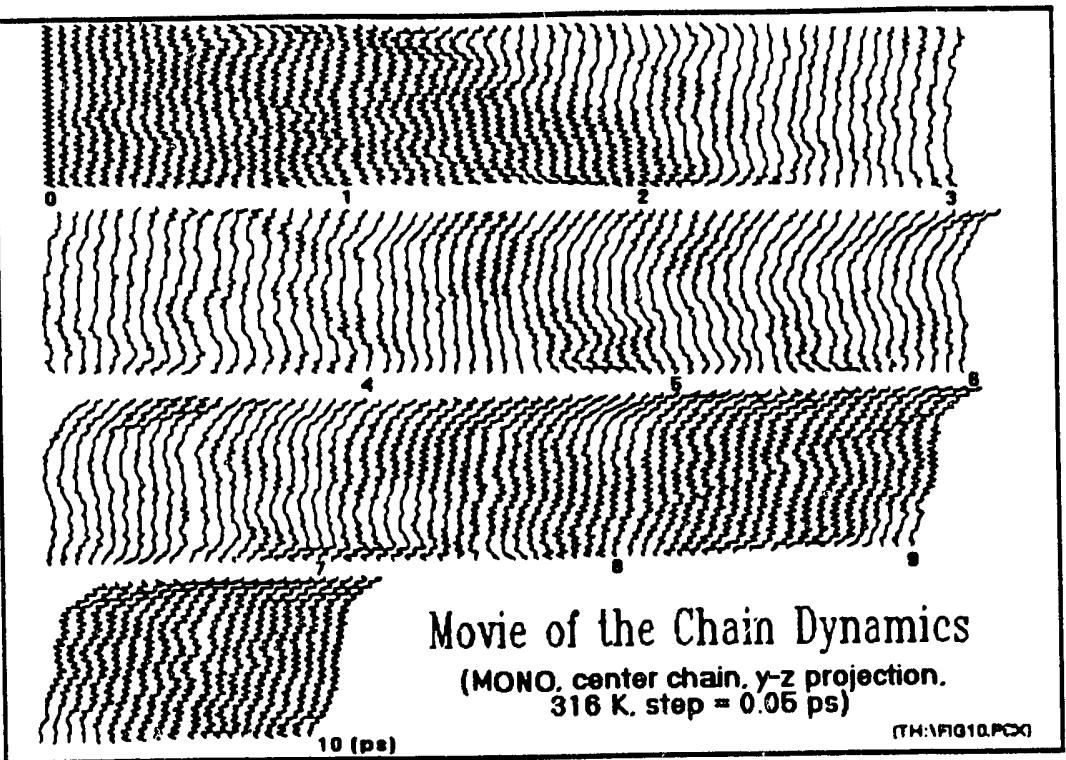

Figure 15 another overall $90^{\circ}$ rotation, turning roughly back to the initial orientation. That the rotation was not a full $180^{\circ}$ turn can be deduced from the direction of the terminal group of the zig-zag chain in Fig. 15. As temperature increases, the frequency and magnitude of this "rotational" motion increases. In addition, the accordion mode of vibration causes diffusion of the whole chain in the crystallographic $c$-direction, and is followed by tilting of the chain-heads.

The next step of analysis was the introduction of an external force to the chain. Adding a free energy gradient on the thermal motion of the crystal permits a diffusion of the chains in the direction of the gradient. To study the mechanism in more detail, the defects were noted in Fig. 16, simultaneously with the positions of the upper and lower chain ends in Fig. 17, and the end-to-end distances in Fig. 18. The illustrated crystal has a chain length of $100 \mathrm{CH}_{2}$ groups. The immediate

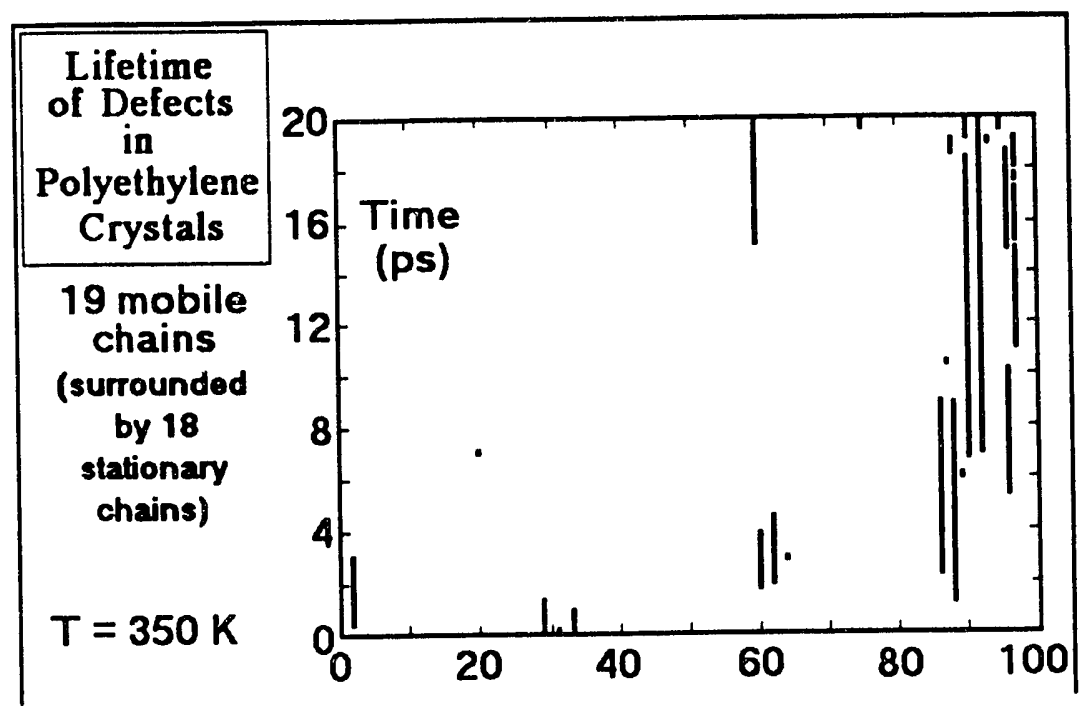
observation is that the gauche defects do not move through the crystal, i.e. they are not involved in the chain diffusion, although without their presence, the transport appears to stop. The actual chain transport must thus be based on gradual twists or accordion-like chain-vibrations, initiated in the gradient direction at the gauche defects. From Fig. 17 it can be seen that the chain moved about $2.5 \mathrm{~nm}$ out of the crystal. The six nearest neighbor positions are marked by the Figure 16

horizontals and have, outside the longitudinal vibration, no translational motion. A move in the chain direction, making use of the amplitude of the longitudinal vibration (longitudinal acoustic vibration, LAM), means a full unit cell motion $(\approx 0.25 \mathrm{~nm})$ would be neces 
sary to maintain crystallographic register with the neighboring chains. Involving the lower frequency torsional oscillationi permits steps of half the unit cell, coupled with a $180^{\circ}$ rotation of the chains. Inspection of Figs. 17 and 18 seems to indicate steps of $c / 2$ superimposed on the LAM, i.e. during the compression phase, the chain end is pulled into the crystal and during the expansion, it is expelled, coupling the LAM with the torsional vibration and involving

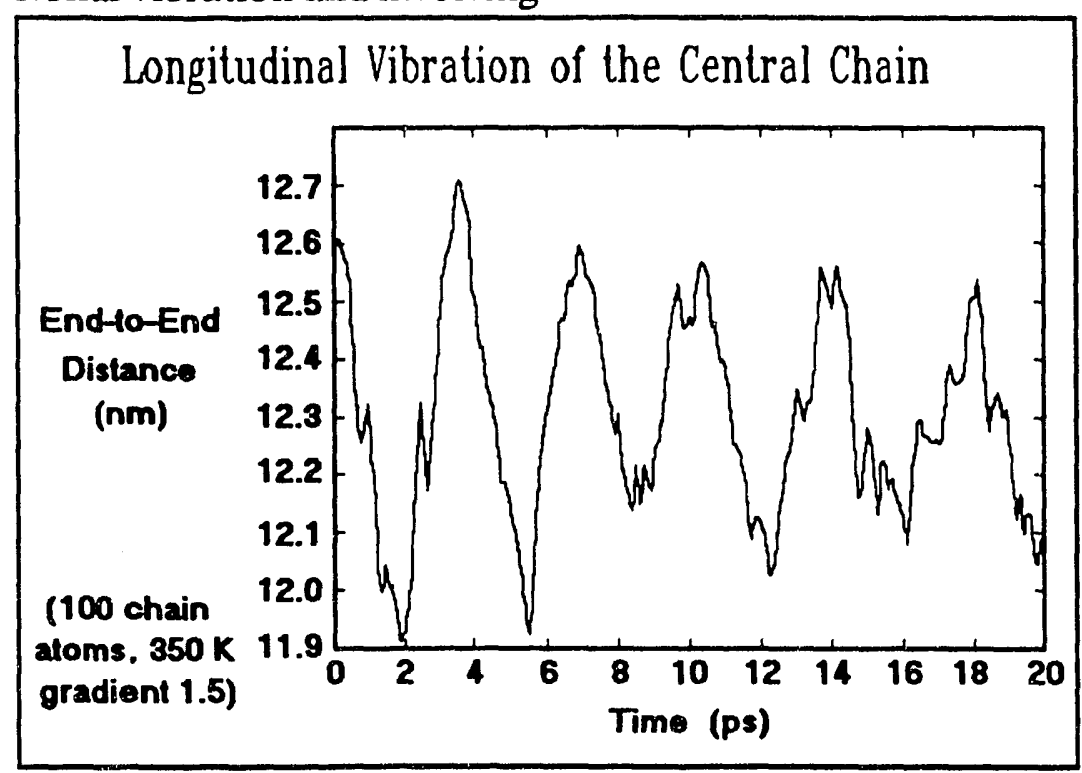

Figure 18

the lamellar crystal, as derived from chain diffusion, is that found for annealing of crystals. Similarly the slowdown of motion with lamellar thickness, as shown in Fig. 20 , is what is expected from experiment.

\section{CONCLLUSIONS}

Many experimental properties of defect crystals are caused by picosecond, largeamplitude motion that can already be simulated by $\mathrm{MD}$

Figure 17

Figure 19

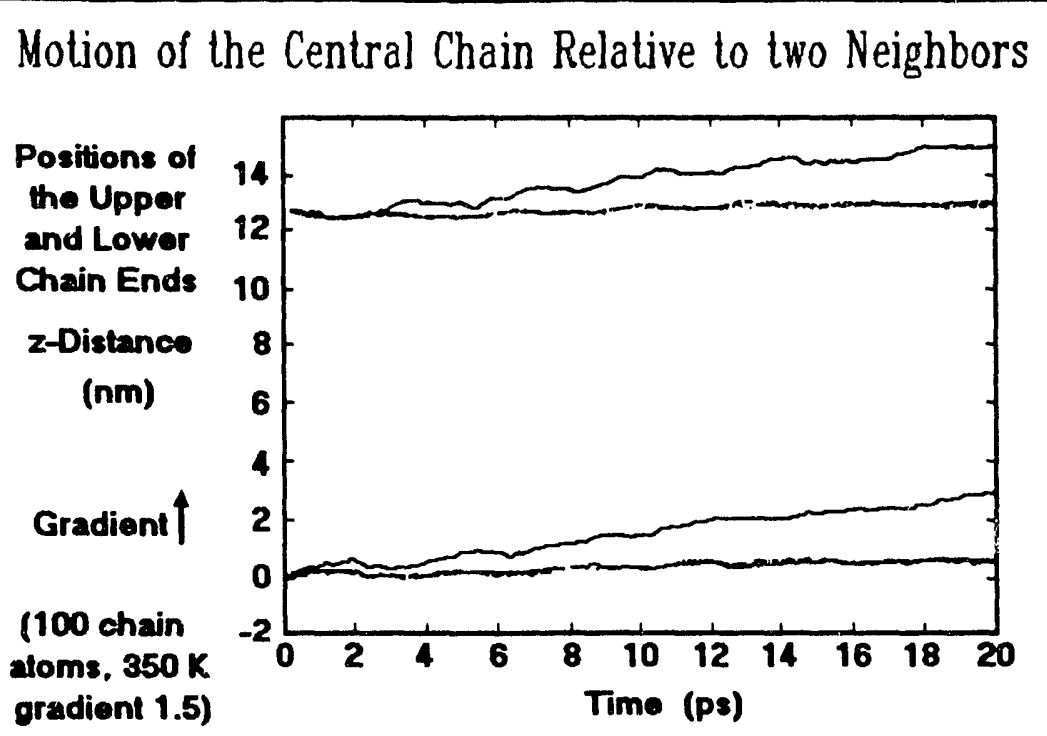

the conformational defect to initiate the motion. During, the approximately 5 vibrations, about $20 \mathrm{c} / 2$-steps occurred, as can be counted from the small irregularities superimposed on the LAM.

Comparison with experimental data shows that the LAM frequency is of the order of magnitude expected, and its change with chain length has the required $1 / \sqrt{n}$ functional relationship. Figure 19 illustrates further, that an increase in thickness of

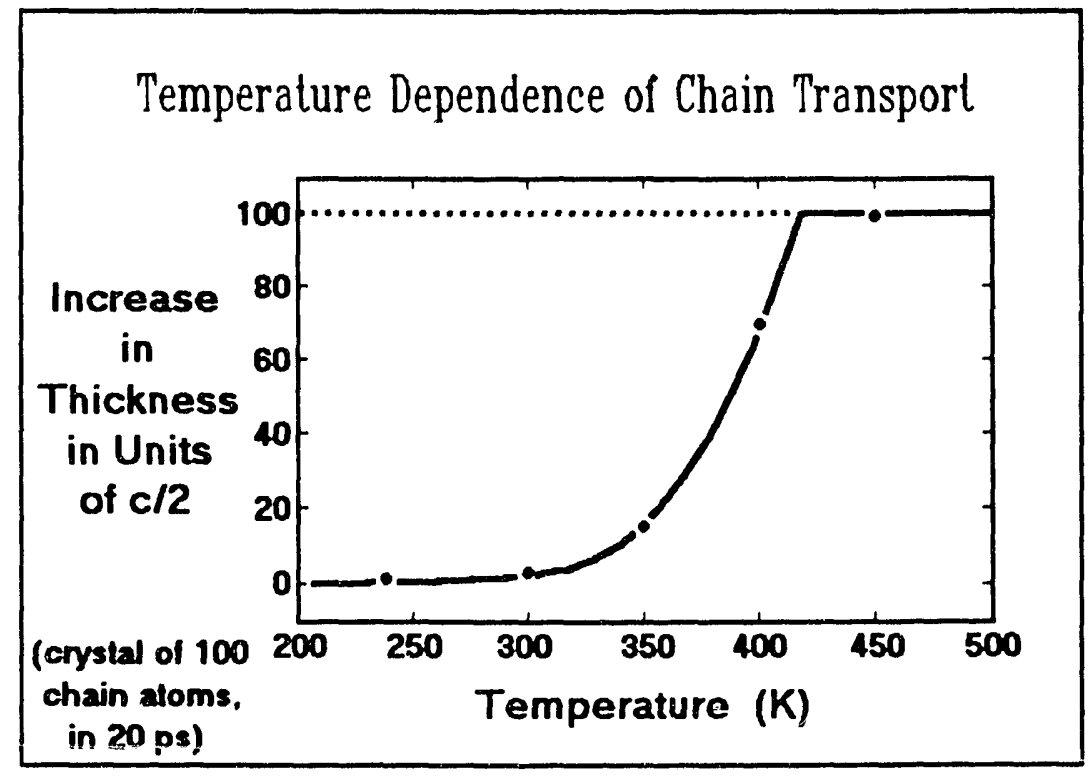


on sufficiently large crystals to give a realistic mechanism. A more definitive picture of the defect solid state of the macromolecules is emerging from this work. The new generation of supercomputers will also permit the solution of problems arising from the inclusion of hydrogen atoms in the simulation, will let us extend the simulations into the nanosecond range, and will solve the many questions involved in the structure and dynamics of the crystal interface.

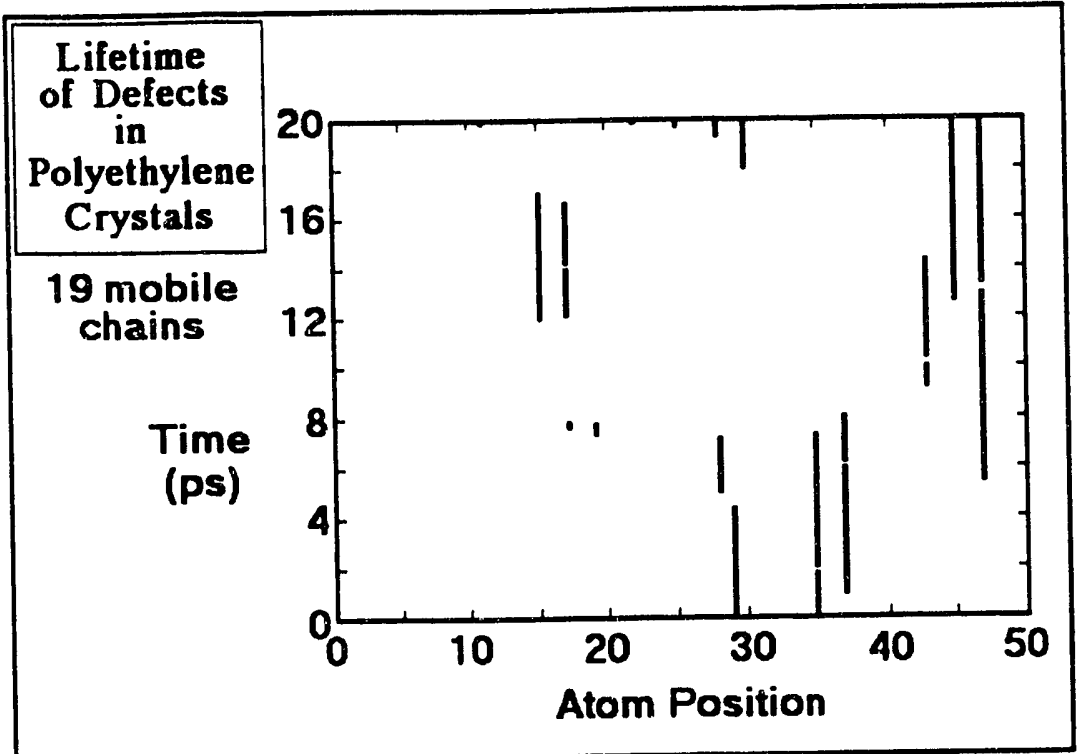

Figure 20

Acknowledgements: This work was supported by the Division of Material Sciences, Office of Basic Energy Sciences, U.S. Department of Energy, under Contract No. DE-AC0584OR21400 with Martin Marietta Energy Systems, Inc., and by the Polymer Program of the National Science Foundation, present Grant No. DMR-92-00520. Computations were performed on the UTCC computers at the University of Tennessee, Knoxville, the Cray XMP at Oak Ridge National Laboratory, the Cray Y-MP at the National Center for Supercomputing Applications, and the Cray Y-MP at the Pittsburgh Supercomputer Center.

\section{$\underline{\text { References: }}$}

1 Sumpter BG, Noid DW, Liang L, Wunderlich, B (1993/4) Atomistic dynamics of macromolecular crystals, Adv Polymer Sci. to be published

2 Noid DW, Sumpter BG, Wunderlich B, Pfeffer GA (1990) Molecular dynamics simulations of polymers: methods for optimal fortran programming, J Comp Chem, 11:236; Noid DW, Sumpter BG, Cox RL (1991) Computational strategies for molecular dynamics simulations of polymer processes: numerical integration schemes, J Comp Polym Sci 1:161

3 Sumpter BG, Noid DW, Wunderlich, B (1990) Computer experiments on the internal dynamics of crystalline polyethylene, mechanistic details of conformational disorder, J Chem Phys 93:6876; (1992) Computational experiments on the motion and generation of defects in polymer crystals, Macromolecules 25:7247

4 Wunderlich B $(1973,1976,1980)$ Macromolecular physics; vol I, crystal structure, morphology, defects; vol II, crystal nucleation, growth, annealing; vol III, crystal melting, Academic Press, New York

5 Kitaigorodskii AI (1955) Organicheskaya Kristallokhimiya, Press of the Akad Sci USSR, Moscow

6 Bunn CW (1939) The crystal structure of long chain normal paraffin hydrocarbons; the "shape" of the $>\mathrm{CH}_{2}$-group, Trans Farad Soc 35:483

7 Wunderlich B (1970) Heat capacities of linear high polymers, Adv Polymer Sci 7:151

8 For an up-to-date copy write to the author

9 Pechhold W (1970) Theorie der Phasenumwandlung in Polymeren, Ber Bunsenges 74:784

10 Reneker DH, Mazur J (1988) Small defects in crystalline polyethylene, Polymer, 29:3 

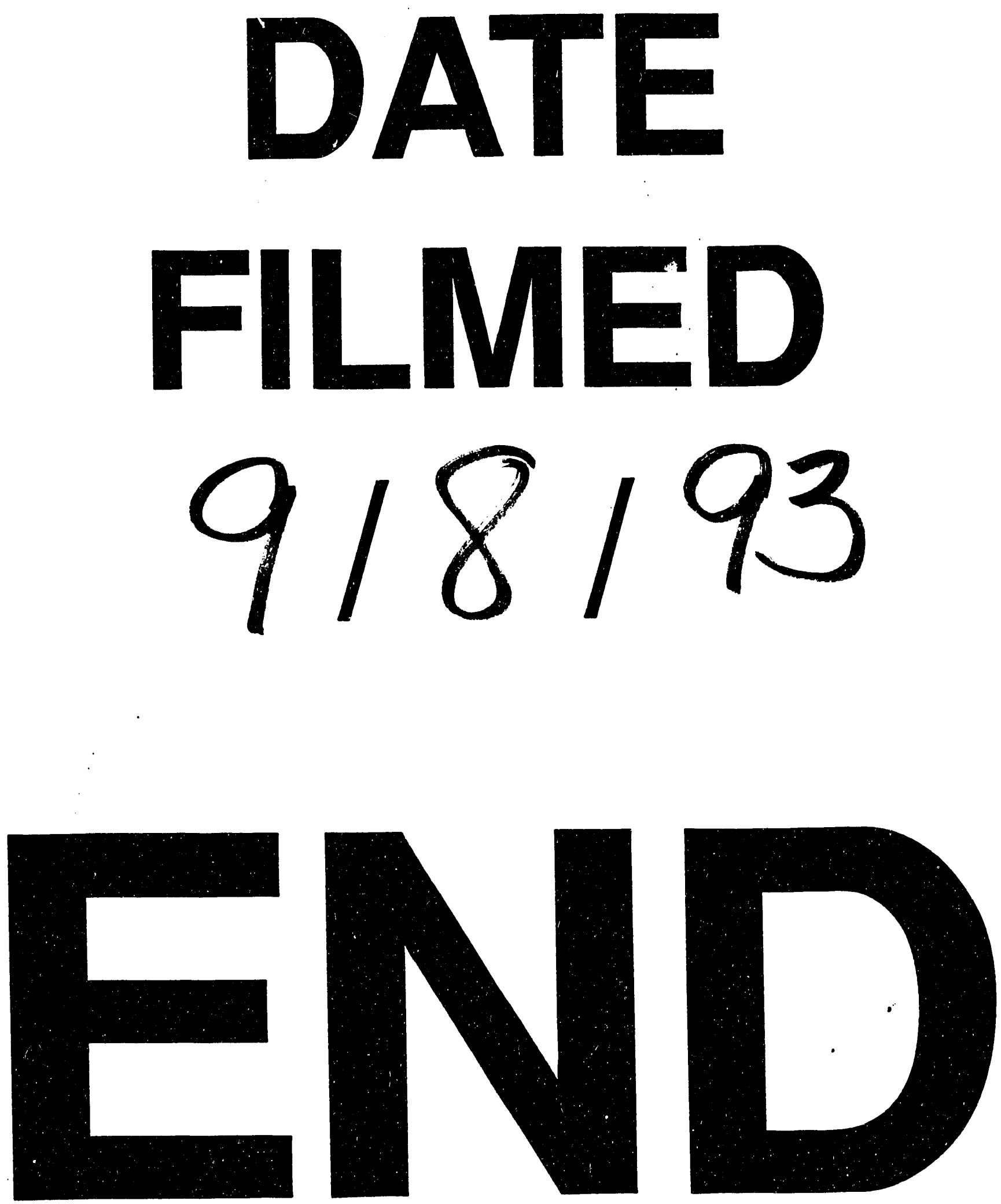
